\title{
Factores del entorno de trabajo que influyen en la ocurrencia de errores de administración de medicación
}

\section{The role of work context factors in medication administration errors}

\author{
L. Ambrosio' ${ }^{1}$, M.J. Pumar-Méndez ${ }^{2}$
}

\section{RESUMEN}

Fundamento. La incidencia de errores de administración de medicación (EAM) es alta y costosa para pacientes e instituciones sanitarias. En su ocurrencia intervienen factores humanos y del entorno de trabajo. El objetivo de este trabajo es identificar los factores del entorno de trabajo que se relacionan con la ocurrencia de EAMs en el ámbito hospitalario.

Metodología. Se llevó a cabo una revisión narrativa de la literatura. Se incluyeron 8 artículos tras revisar las bases de datos MEDLINE, CINAHL y COCHRANE LIBRARY, durante el periodo 2002-2012.

Resultados. Las distracciones e interrupciones, la sobrecarga de trabajo, el diseño de las unidades y las características del material han sido destacados entre los factores del entorno de trabajo que intervienen en la ocurrencia de errores de administración de medicación.

Conclusiones. La creación de artefactos organizacionales para reducir las interrupciones; la implicación del paciente en la administración de medicación; la introducción de nuevas tecnologías y la mejora del etiquetado de los medicamentos puede ayudar a reducir la incidencia de errores de administración de medicación. Para avanzar en la identificación y gestión de factores precursores de errores de administración de medicación se recomienda la realización de estudios de cohortes prospectivas o revisiones.

Palabras clave. Seguridad del paciente. Error de medicación. Administración de medicación. Entorno de las instalaciones sanitarias. \begin{abstract}
Background. The incidence of medication administration errors (MAE) is high and costly for patients and health institutions. Human factors and factors relating to the work context intervene in their occurrence. The aim of this article is to identify the role of factors in the work setting related to occurrence of MAEs in the hospital field.
\end{abstract}

Methods. A narrative review of the literature. Eight articles were included following a review of MEDLINE, CINAHL and COCHRANE LIBRARY databases for the 2002-2012 period.

Results. Distractions and interruptions, work overload, design of units and characteristics of the material stood out amongst the work context factors intervening in the occurrence of medication administration errors.

Conclusions. The creation of organizational artefacts to reduce interruptions; patient involvement in medication administration; the introduction of new technologies and improving the labelling of medications can help reduce the incidence of medication administration errors. To advance in identifying and managing factors that are precursors of medication administration errors, we recommend the carrying out of studies or reviews of prospective cohorts.

Key words. Patient safety. Medication error. Medication administration. Health installation context.
1. Dpto. Enfermería de la Persona Adulta. Facultad de Enfermería de la Universidad de Navarra. Pamplona

2. Dpto. Enfermería Comunitaria y Materno-Infantil. Facultad de Enfermería de la Universidad de Navarra. Pamplona

Recepción: 1 de octubre de 2012

Aceptación provisional: 16 de noviembre de 2012

Aceptación definitiva: 26 de noviembre de 2012

\section{Correspondencia:}

Dña. Leire Ambrosio Gutiérrrez

Departamento de Enfermería de la Persona

Adulta

Facultad de Enfermería

Universidad de Navarra

31008 Pamplona

lambrosio@unav.es 


\section{INTRODUCCIÓN}

El término error de medicación hace referencia a aquellos fallos no intencionales durante el proceso de medicación, que tienen el potencial de producir daño en el paciente ${ }^{1,2}$. En el ámbito internacional se ha estimado que el $10-20 \%$ de los eventos adversos que ocurren en el ámbito hospitalario corresponden a errores de medicación ${ }^{3}$. En España, según los datos del Estudio Nacional sobre los Efectos Adversos ligados a la hospitalización (ENEAS), el 4\% de los pacientes ingresados en hospitales experimentan evento adversos relacionados con la medicación, que en un $34,8 \%$ de los casos son evitables ${ }^{4}$.

El proceso de medicación se compone de cuatro subprocesos: prescripción, transcripción, dispensación y administración de la medicación. Los fallos no intencionados durante el subproceso de administración de medicación, o errores de administración de medicación (EAM), constituyen la segunda causa más frecuente por la que ocurren los errores de medicación ${ }^{5}$. Más concretamente, se estima que suponen un $49 \%$ de los errores de medicación ${ }^{6}$.

Los eventos adversos relacionados con la medicación son causa importante de morbilidad, mortalidad, prolongación de estancias hospitalarias, reingresos y aumento del gasto sanitario ${ }^{7,9}$. No en vano el coste global para el sistema de salud español de los efectos adversos relacionados con la medicación se ha cifrado entre los 469 y 790 millones de euros anuales ${ }^{10}$. Teniendo en cuenta el calado del problema, disminuir los errores de medicación y concretamente los EAM, es una prioridad para instituciones sanitarias y profesionales de la salud ${ }^{11,12}$. Dentro de este último grupo de interés, los profesionales de enfermería tienen un papel especialmente relevante, ya que la administración de medicación es una de sus responsabilidades en la que llegan a invertir hasta un $40 \%$ de su tiempo ${ }^{13}$.

La investigación existente sobre la causalidad de los errores de medicación revela que éstos son generalmente resultado de múltiples fallos que a su vez pueden tener distinto origen ${ }^{7,14}$. Es común que entre las causas de un error de medicación se conjuguen fallos activos y fallos latentes. Los fallos activos se refieren a errores por comisión u omisión ejecutados por los profesionales a pie de cama, como pueden ser olvidos o imprecisiones en la realización de procedimientos. Los fallos latentes se refieren a errores en el diseño organizativo como, por ejemplo, inadecuaciones en la distribución de las unidades de trabajo o en la disposición de recursos humanos. Este último tipo de fallos son peligrosos, no sólo porque pueden provocar errores de modo directo, sino porque además, crean condiciones desfavorables para trabajar, en las que los profesionales son más proclives a errar. Asímismo, la peligrosidad de los fallos latentes radica en que al ser menos obvios que los fallos activos pueden pasar desapercibidos para los gestores, recibiendo escasa atención y perpetuándose en el tiempo ${ }^{15}$.

La actual filosofía para la gestión de riesgo en el ámbito clínico se centra en la detección y corrección de fallos latentes ${ }^{14,16,17}$. Este enfoque se justifica no sólo en base a la gran peligrosidad que acarrean, sino también teniendo en cuenta que éstos son más previsibles y modificables que los fallos activos. De hecho, investigaciones llevadas a cabo en el campo de la psicología, han demostrado la naturaleza falible del ser humano y, en consecuencia, la inevitabilidad e imprevisibilidad de la ocurrencia de fallos activos ${ }^{18}$.

Entre los fallos latentes se pueden clasificar los fallos en el diseño del entorno de trabajo. El término entorno de trabajo hace referencia al conjunto de elementos relativamente permanentes que rodean el ámbito en el que trabajan los profesionales sanitarios ${ }^{19}$. Los expertos en la gestión de riesgos apuntan que para reducir la incidencia de EAMs es imprescindible mejorar los entornos de trabajo ${ }^{20-22}$. Para poder intervenir de modo efectivo en esta dirección, es preciso conocer cuáles son los factores del entorno de trabajo que se relacionan con la ocurrencia de EAM. Con este objetivo se ha llevado a cabo una revisión de la literatura. 


\section{MÉTODOS}

Se llevó a cabo una revisión narrativa de la literatura, con el objetivo de identificar los factores del entorno de trabajo del hospital que influyen en la ocurrencia de EAM. La revisión se realizó en las bases de datos del ámbito sanitario más relevantes para el tema: MEDLINE (PubMed), CINAHL (EBSCO) y COCHRANE LIBRARY, combinan- do los términos que se recogen en la figura 1. Con el objetivo de que la revisión fuera actual, la búsqueda fue limitada a estudios publicados entre 2002 y 2012 en lengua española e inglesa. Para complementar la búsqueda en las bases de datos electrónicas, e identificar estudios adicionales que fueran de relevancia, se revisaron las listas de referencias bibliográficas de los artículos seleccionados.

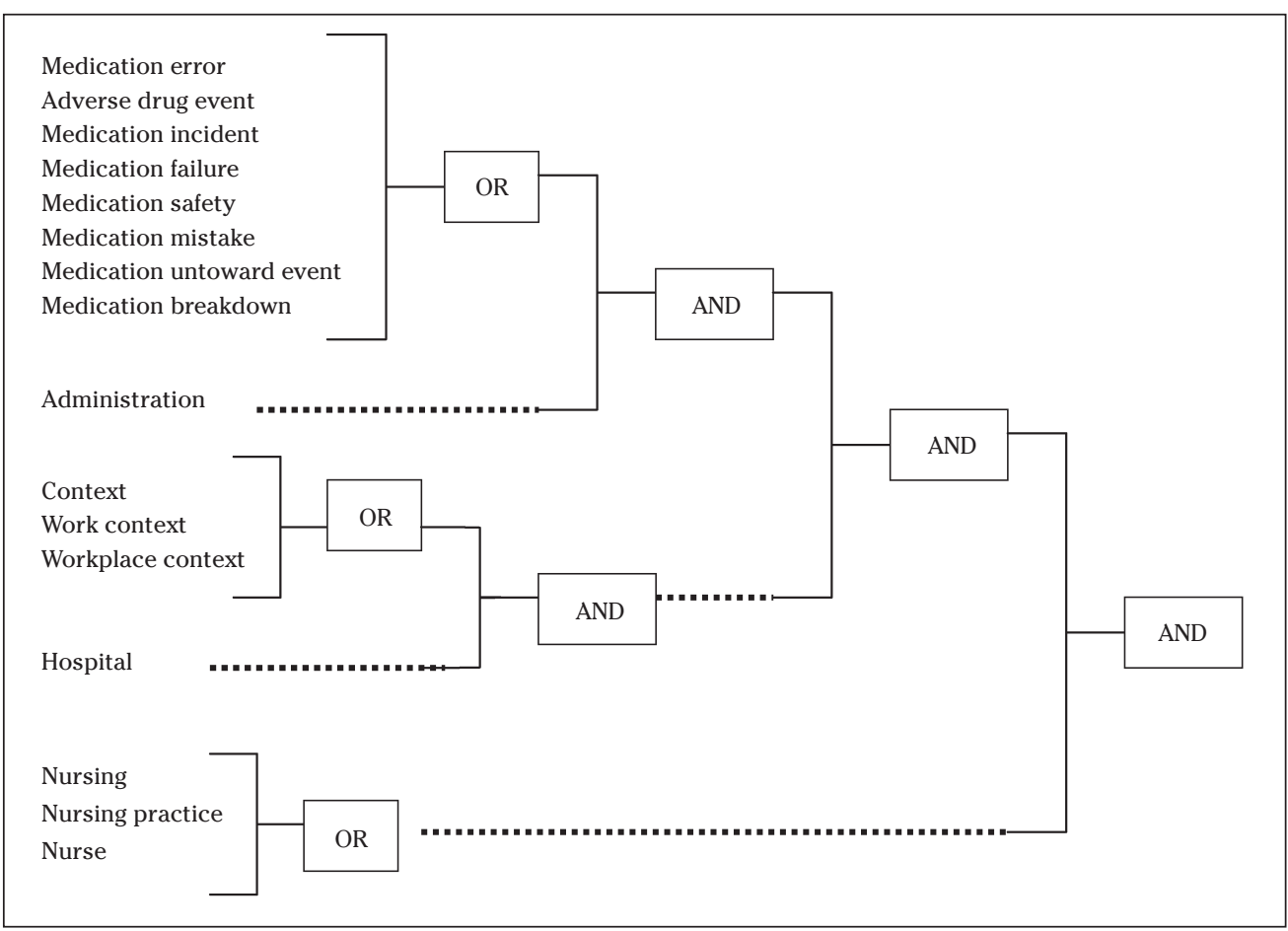

Figura 1. Términos clave introducidos en las bases de datos electrónicas.

La selección de estudios para la revisión incluyó la lectura, por parte del primer autor de este artículo, de todos los títulos y resúmenes de los registros identificados en las búsquedas realizadas en bases de datos electrónicas, reteniendo únicamente aquellos potencialmente relevantes para responder al objetivo de la revisión. De estos últimos, se obtuvo el texto completo, y los dos autores del articulo evaluaron su elegibilidad en base a criterios de inclusión y exclusión establecidos con anterioridad y que se exponen en la tabla 1. 
Tabla 1. Criterios de selección de los artículos

\section{Criterios de inclusión}

Estudios sobre EAM, en los que estaban involucrados profesionales de enfermería, y que se llevaron a cabo en el ámbito hospitalario
Criterios de exclusión

Estudios que traten sobre errores de medicación de manera genérica
Estudios que tengan por objetivo identificar factores del entorno de trabajo que influyen en la ocurrencia de EAM tanto percibidos como objetivados
Estudios no realizados con profesionales de enfermería o fuera del ámbito hospitalario
Estudios cuya metodología sea apropiada para dar respuesta al objetivo de la presente revisión:

- Análisis retrospectivos de historias clínicas

- Estudios prospectivos de cohortes

- Revisiones de la literatura

- Estudios cualitativos

- Estudios con metodología combinada
Estudios que no aporten datos empíricos: opiniones, literatura gris (excepto tesis doctorales).

\section{RESULTADOS}

Se identificaron un total de 531 registros. Tras la lectura del título o resumen, 503 fueron descartados por no mantener relación con el tema abordado. De los artículos restantes 20 fueron excluidos por no cumplir los criterios de inclusión. Las características de los 8 estudios seleccionados para la revisión se detallan en la tabla 2.

Como se puede observar en la tabla 2 , se identificaron diversos factores del entorno de trabajo hospitalario precursores de la ocurrencia de EAM. En concreto, son tres los factores del entorno de trabajo que se citaron con más frecuencia: las distracciones, las interrupciones, y la sobrecarga de trabajo ${ }^{13,20,21,23-27}$. Otros factores como son el diseño de la unidad de trabajo y las características del material, también han sido identificados como factores influyentes en la ocurrencia de EAM, aunque en un menor número de estudios ${ }^{20,21,24-26}$.

\section{Distracciones e interrupciones}

En seis de los ocho estudios incluidos en la presente revisión ${ }^{13,21,23-26}$, se identifi- caron las distracciones e interrupciones como factores del entorno de trabajo que influyen en la ocurrencia de EAM. Además, en 5 estudios ${ }^{21,25,28-30}$, se señaló que las distracciones e interrupciones son la causa más frecuente de los EAM.

El término interrupción es definido como una situación en la que un profesional deja de realizar una actividad para abordar una cuestión externa ${ }^{21,31}$. Por su parte, el término distracción se refiere al acto de dejar de realizar una acción por sentirse atraído por otra ${ }^{32,33}$. Distracciones e interrupciones son conceptos diferentes, aunque íntimamente relacionados, ya que, por ejemplo, las interrupciones pueden ser causa de distracciones ${ }^{21}$.

En los estudios seleccionados se describieron circunstancias y factores del entorno de trabajo que influyen en la ocurrencia de distracciones e interrupciones durante la administración de medicación. Los reclamos por parte de los pacientes y las llamadas telefónicas son las dos más mencionadas ${ }^{25,28}$, seguidas de la ausencia de medicamentos y de material en el momento de la administración de medicación ${ }^{21,23-25}$. 
Tabla 2. Características principales de los artículos seleccionados

\begin{tabular}{|c|c|c|c|}
\hline Referencia & Objetivo & $\begin{array}{l}\text { Diseño/Métodos/ } \\
\text { Contexto del estudio }\end{array}$ & $\begin{array}{l}\text { Factores del entorno del } \\
\text { trabajo identificados }\end{array}$ \\
\hline $\begin{array}{l}\text { Armitage \& } \\
\text { Knapman } \\
(2003)^{13}\end{array}$ & $\begin{array}{l}\text { Identificar los factores que } \\
\text { influyen en la ocurrencia de } \\
\text { EAM }\end{array}$ & $\begin{array}{l}\text { Revisión narrativa } \\
\text { Incluye estudios en } \\
\text { hospitales en Reino Unido }\end{array}$ & $\begin{array}{l}\text { - Sobrecarga de trabajo } \\
\text { - Distracciones e interrup- } \\
\text { ciones }\end{array}$ \\
\hline $\begin{array}{l}\text { Bennet, } \\
\text { Dawoud \& } \\
\text { Maben }(2010)^{23}\end{array}$ & $\begin{array}{l}\text { Examinar cómo las } \\
\text { interrupciones influyen } \\
\text { en la ocurrencia de EAM e } \\
\text { identificar estrategias para } \\
\text { disminuir las interrupciones } \\
\end{array}$ & $\begin{array}{l}\text { Revisión narrativa } \\
\text { Incluye estudios en } \\
\text { hospitales en Reino Unido }\end{array}$ & $\begin{array}{l}\text { - Distracciones e interrup- } \\
\text { ciones }\end{array}$ \\
\hline $\begin{array}{l}\text { Carlton \& } \\
\text { Blegen }(2006)^{20}\end{array}$ & $\begin{array}{l}\text { Evaluar la incidencia de los } \\
\text { EAM y explorar sus factores } \\
\text { precursores }\end{array}$ & $\begin{array}{l}\text { Revisión narrativa } \\
\text { No especifica procedencia de } \\
\text { los estudios }\end{array}$ & $\begin{array}{l}\text { - Sobrecarga de trabajo } \\
\text { - Características de la unidad } \\
\text { de trabajo }\end{array}$ \\
\hline $\begin{array}{l}\text { Esi Owusu \& } \\
\text { While }(2010)^{24}\end{array}$ & $\begin{array}{l}\text { Explorar los factores } \\
\text { humanos y organizacionales } \\
\text { que contribuyen a } \\
\text { los EAM precipitados } \\
\text { por profesionales de } \\
\text { enfermería en unidades de } \\
\text { hospitalización } \\
\end{array}$ & $\begin{array}{l}\text { Revisión narrativa } \\
\text { Incluye estudios en } \\
\text { hospitales en Reino Unido }\end{array}$ & $\begin{array}{l}\text { - Distracciones e interrup- } \\
\text { ciones } \\
\text { - Sobrecarga de trabajo } \\
\text { - Diseño del material }\end{array}$ \\
\hline $\begin{array}{l}\text { Fry \& Dacey } \\
(2007)^{25}\end{array}$ & $\begin{array}{l}\text { Explorar los factores que } \\
\text { contribuyen a la ocurrencia } \\
\text { de EAM }\end{array}$ & $\begin{array}{l}\text { Revisión narrativa } \\
\text { Incluye estudios en } \\
\text { hospitales en Reino Unido }\end{array}$ & $\begin{array}{l}\text { - Sobrecarga de trabajo } \\
\text { - Distracciones e interrup- } \\
\text { ciones } \\
\text { - Diseño y características del } \\
\text { material }\end{array}$ \\
\hline $\begin{array}{l}\text { McBridge- } \\
\text { Henry \& } \\
\text { Foureur (2006) } \\
{ }_{26}\end{array}$ & $\begin{array}{l}\text { Identificar los factores que } \\
\text { influyen en la ocurrencia de } \\
\text { EAM y examinar el rol de } \\
\text { enfermería en la prevención } \\
\text { de éste tipo de errores }\end{array}$ & $\begin{array}{l}\text { Revisión narrativa } \\
\text { Incluye estudios en } \\
\text { hospitales en Nueva Zelanda }\end{array}$ & $\begin{array}{l}\text { - Falta de personal adecuado } \\
\text { - Falta de protocolos } \\
\text { - Iluminación, ubicación del } \\
\text { almacén } \\
\text { - Sobrecarga de trabajo } \\
\text { - Distracciones }\end{array}$ \\
\hline $\begin{array}{l}\text { Popescu, } \\
\text { Currey \& Botti } \\
(2011)^{21}\end{array}$ & $\begin{array}{l}\text { Explorar los diferentes } \\
\text { factores del entorno que } \\
\text { influyen en la ocurrencia de } \\
\text { EAM }\end{array}$ & $\begin{array}{l}\text { Estudio cualitativo } \\
\text { exploratorio descriptivo } \\
\text { (observaciones y entrevistas) } \\
\text { Hospital Australiano }\end{array}$ & $\begin{array}{l}\text { - Distracciones e interrup- } \\
\text { ciones } \\
\text { - Ubicación del almacén } \\
\text { - Diseño de las unidades de } \\
\text { trabajo }\end{array}$ \\
\hline $\begin{array}{l}\text { Tissot y col } \\
(2003)^{27}\end{array}$ & $\begin{array}{l}\text { Evaluar la incidencia de } \\
\text { EAM en la práctica clínica } \\
\text { e identificar los factores de } \\
\text { riesgo que influyen en la } \\
\text { ocurrencia de EAM }\end{array}$ & $\begin{array}{l}\text { Metodología combinada } \\
\text { (observaciones y análisis } \\
\text { cuantitativo) } \\
\text { Hospital universitario francés }\end{array}$ & $\begin{array}{l}\text { - Falta de protocolos } \\
\text { - Sobrecarga de trabajo }\end{array}$ \\
\hline
\end{tabular}

\section{Sobrecarga de trabajo}

La sobrecarga de trabajo fue identificada en siete de los ocho estudios seleccionados para la presente revisión ${ }^{13,20,21,24-27}$ siendo señalado como la segunda causa por la que se precipitan los EAM.

El término sobrecarga de trabajo hace referencia a un exceso de requerimientos físicos y psicológicos a los que se ven sometidos los profesionales a lo largo de su jornada laboral ${ }^{34}$. Los estudios exponen que este factor influye de forma directa e indirecta en la ocurrencia de EAM ${ }^{20,24,26}$. Por un lado, la sobrecarga de trabajo se ha relacionado directamente con la ocurrencia de fallos activos precipitantes de los EAM. En 
segundo lugar, los estudios sugieren que la sobrecarga de trabajo influye indirectamente en la ocurrencia de EAM provocando condiciones que potencian la capacidad humana de errar (como son el estrés y cansancio), y fomentando la comisión de violaciones de protocolos de administración segura de medicación. Con respecto a este último punto, la literatura especifica que la regla de los "5 correctos" y los procedimientos de "doble revisión" son los aspectos más obviados de los protocolos de administración segura de medicación ${ }^{13,24-26}$.

\section{Diseño de las unidades de trabajo}

El diseño de las unidades de trabajo se refiere al conjunto de elementos que conforman la estructura física del entorno de trabajo $^{21}$. La inadecuación de dos elementos del diseño de las unidades de trabajo han sido identificadas recurrentemente en la literatura como factores potencialmente influyentes en la ocurrencia de EAM: la iluminación del espacio de trabajo y la ubicación de los almacenes de material ${ }^{21,26,33,35,36}$. Por ejemplo, la iluminación deficiente del espacio de trabajo puede generar problemas para apreciar el etiquetado de la medicación que acaben desencadenando confusiones precipitantes de $\mathrm{EAM}^{26,35}$. Así mismo, los hallazgos de dos estudios ${ }^{33,36}$ sugieren que mantener el almacén de medicación apartado del lugar de la administración de medicación aumenta la ocasión de errar, debido a que en los desplazamientos se genera la oportunidad para un mayor número de interrupciones y distracciones de los profesionales de enfermería.

\section{Características del material}

La categoría "características del material" hace referencia a diversas características de los medicamentos que pueden dar lugar a dificultades a la hora de ser administrarlos, como son: el etiquetado, envasado o nomenclatura indiferenciada o similar entre medicamentos o los mecanismos de administración poco comunes o innovadores ${ }^{24,25,28}$. Estas características del material tienen la potencialidad de generar ambigüedad y confusiones en la vía, dosis (cantidad y concentración), preparación y método de administración de los medicamentos, conducentes a los $\mathrm{EAM}^{28}$.

\section{DISCUSIÓN}

Los hallazgos de la presente revisión revelan que los factores del entorno del trabajo que intervienen en la ocurrencia de EAM son múltiples. Las distracciones, interrupciones y la sobrecarga de trabajo son los más señalados en la literatura ${ }^{13,23-26}$.

Las distracciones e interrupciones en el entorno de trabajo no pueden ser eliminadas por completo, pero sí pueden ser reducidas $^{37}$. La literatura propone varias estrategias para alcanzar este objetivo, como son: la implementación de áreas de silencio durante la administración de medicación $^{38,39}$; el uso de carteles en las unidades de trabajo que incluyan mensajes dirigidos a evitar las distracciones e interrupciones $^{32}$; o el uso de chalecos que remarquen el momento de administración de medicación y la necesidad de que las enfermeras no sean interrumpidas en ese momento ${ }^{23}$. La comparabilidad de los estudios en los que se han testado estas intervenciones es limitada dado que se han llevado a cabo en contextos muy dispares. Por ello, es difícil llegar a conclusiones sobre cuál es la intervención más efectiva. Valorar la viabilidad de implantar las distintas estrategias en el contexto hospitalario español tampoco es sencillo, ya que todos los estudios se han llevado a cabo fuera del ámbito nacional. Sin embargo, es previsible que ciertos problemas que han surgido en otros contextos surjan también en el nuestro, como por ejemplo, la falta de espacio para implementar áreas de silencio en los hospitales o la falta de concienciación de los profesionales de enfermería sobre la peligrosidad de las interrupciones y distracciones durante la administración de medicación. Teniendo en cuenta estas premisas para la implantación, las estrategias basadas la creación de artefactos organizacionales para que no se interrumpa el proceso de administración de medicación, como la introducción de 
carteles o chalecos, parecen opciones más viables a corto plazo.

La sobrecarga de trabajo es otro de los factores del entorno de trabajo identificado en la presente revisión como factor influyente en la ocurrencia de EAM. La literatura $^{13,20,21,24-27}$ sugiere que la sobrecarga de trabajo puede generar tanto fallos activos tales como olvidos o inadvertencias de los profesionales, como condiciones que potencian la falibilidad humana y en consecuencia la ocurrencia de EAM, entre las que destacan el estrés y el cansancio de los profesionales ${ }^{20,24,26}$. Asímismo, la sobrecarga de trabajo se ha asociado a otro potencial precipitante de los EAM: la violación de protocolos de administración segura de medicación. Entre las medidas descritas en la literatura para reducir estas violaciones destacan: la estandarización de los horarios de administración de medicación; la individualización de la preparación y administración de medicación (es decir, sin acumular preparaciones de varios pacientes $\left.^{21,24,26}\right)$; y la implicación del paciente en la administración de la medicación ${ }^{20,21,24,25}$. Esta última medida requiere educar previamente al paciente en el tipo de medicación que toma de modo que pueda participar activamente junto con la enfermera en la comprobación de la medicación que se le va a administrar. De esta manera, durante el proceso de administración de medicación se garantiza el cumplimiento de los dos aspectos más obviados de los protocolos de administración segura de medicación: la "doble revisión" y la comprobación de los "5 correctos".

La incorporación de nuevas tecnologías, como los lectores y escáneres de código de barras, que automatizan el proceso de administración de medicación es otra medida que puede facilitar el cumplimiento de protocolos de administración segura de medicación $\mathrm{n}^{40-42}$. Estas tecnologías agilizan la comprobación de la identidad del paciente y de la adecuación de la medicación ${ }^{40}$. Aunque ya existe evidencia sobre la eficacia de estas tecnologías, hay que tener en cuenta que su incorporación supone una importante inversión económica, que en el contexto económico actual puede parecer inviable ${ }^{43}$. Sin embargo, si se tiene en cuenta el alto coste económico de los EAM, la introducción de sistemas que los reduzcan puede suponer un ahorro económico a largo plazo. Las evaluaciones de la costo-efectividad del uso de estas nuevas tecnologías puede ser necesaria para hacer explícito su valor ante los gestores.

El diseño de las unidades de trabajo y las características del material son otros de los factores identificados como posibles precursores de los EAM $^{24,25}$. La literatura propone estrategias para distinguir medicamentos y evitar confusiones como: la realización de cambios en el diseño del envoltorio o el uso de etiquetas de colores para diferenciar medicamentos que presentan una nomenclatura, etiquetado o envasado similares; la especificación en el etiquetado de todas las características del medicamento ${ }^{44-46}$; el establecimiento de carteles de alerta en los almacenes; o la creación de listados farmacéuticos de aquellos medicamentos que pueden dar lugar a errores por sus características similares $^{21,44}$. Estas intervenciones suponen un coste más modesto que otro tipo de intervenciones, como la introducción de nuevas tecnologías por lo que pueden ser más fácilmente adoptadas a corto plazo.

A las estrategias para la prevención de EAM expuestas anteriormente, es necesario añadir la creación de sistemas de notificación y análisis de errores. Analizando los errores que notifican tanto profesionales como pacientes, se pueden identificar factores del entorno de trabajo (además de factores de otra índole) que contribuyen a la ocurrencia de errores y valorar cuáles son las medidas preventivas más pertinentes para solventar los mismos ${ }^{7,47,48}$. La implantación de programas de notificación de errores en el ámbito hospitalario español requiere el desarrollo de una cultura de seguridad que fomente el enfoque no punitivo en la gestión de riesgos y el aprendizaje organizacional $^{49}$.

Los resultados de esta revisión deben ser interpretados con cierta cautela ya que los estudios incluidos ${ }^{13,20,21,23-27}$ no están exentos de limitaciones. Por ejemplo, en varios estudios el objetivo no está bien de- 
finido ${ }^{13,20,23-25}$ mientras que en otros ${ }^{21,27}$ no se describe con suficiente detalle los métodos empleados para la recogida o análisis de los datos. Estas limitaciones impiden evaluar el rigor de los resultados obtenidos en los estudios. Por otro lado hay que tener en cuenta que la mayoría de los estudios se limitan a una sola institución de características particulares lo que limita la posibilidad de generalización de resultados.

Además de las limitaciones de la literatura, la presente revisión también cuenta con ciertas limitaciones. No en vano, la revisión de la literatura llevada a cabo se trata de una revisión narrativa. Este tipo de revisiones pueden ofrecer una visión amplia de la temática abordada pero no pueden ser consideradas como exhaustivas ${ }^{50}$. Por tanto, es posible que se hayan podido omitir estudios relevantes ${ }^{51,52}$.

Por su alto coste humano y económico la prevención de EAM es un imperativo ético para los profesionales e instituciones sanitarias. Por ello, la mejora de los entornos de trabajo debe ser un objetivo prioritario para gestores y ejecutivos de dichas instituciones.

\section{BIBLIOGRAFÍA}

1. Ferner RE, Aronson JK. Clarification of terminology in medication errors: definitions and classification. Drug Saf 2006; 29: 1011-1022.

2. Brady AM, Malone AM, Fleming S. A literature review of the individual and systems factors that contribute to medication errors in nursing practice. J Nurs Manag 2009; 17: 679-697.

3. OTERo MJ. Errores de medicación y gestión de riesgos. Rev Esp Salud Pública 2003; 77: 527-540.

4. Estudio Nacional sobre los Efectos Adversos ligados a la hospitalización: ENEAS 2005. Ministerio de Sanidad y Consumo. Madrid: 2006.

5. CLARK PA. Medication errors in family practice, in hospitals and after discharge from the hospital: an ethical analysis. J Law Med Ethics 2004; 32: 349-357.

6. National Patient Safety Agency [revista electrónica], 2012. [Consultado 04-06-2012]: Disponible en http://www.npsa.nhs.uk.

7. Kohn LT, Corrigan JM, DonaldSon MS. To Err is Human: Building a Safer Health System Institute of Medicine. National Academy Press, 1999.
8. Otero MJ, Alonso P, Martín R, Valverde MP, Domínguez A. Analysis of preventable adverse drug events (ADEs) leading to hospital admission: incidence, categorization and cost. 36th ASHP Midyear Clinical Meeting and Exhibits, December 2-6, 2001. New Orleans (LA).

9. Dennison RD. Creating an organizational culture for medication safety. Nurs Clin North Am 2005; 40: 1-23.

10. Revisión Bibliográfica sobre Trabajo de Costes de la "No Seguridad del Paciente". Ministerio de Sanidad y Consumo. Madrid: 2008.

11. Australian Council for Safety and Quality in Healthcare. Second national report on patient safety: improving medication safety 2002. [Consultado 04-06-2012]. Disponible en http://www.safetyandquality.gov.au.

12. Stetina P, Groves M, Pafford L. Managing medication errors: A qualitative study. Medsurg Nurs 2005; 14: 174-178.

13. Armitage G, Knapham H. Adverse events in drug administration: a literature review. J Nurs ManaG 2003; 11: 130-140.

14. REASON JT. Understanding adverse events: human factors. Qual Health Care 1995; 4: 31-54.

15. REASON JT. Human error: models and management. BMJ 2000; 320: 768-796.

16. REASON JT. Human error. Cambridge University Press, 1990.

17. Vincent C, Stanhope N, Crowley-Murphy M. Reasons for not reporting adverse events: an empirical study. J Eval Clin Pract 1999; 5: 13-21.

18. Norman DA. The Psychology of everyday things. Basic Books, 1998

19. Kelloway EK, DAY AL. Building healthy workplaces: What we know so far. Can J Behavioural Science 2005; 37: 223-235.

20. Carlton G, Blegen MA. Medication-related errors: a literature review of incidence and antecedents. Annu Rev Nurs Res 2006; 24: 1938.

21. Popescu A, Currey J, Botti M. Multifactorial Influences on and Deviations from Medication Administration Safety and Quality in the Acute Medical/Surgical Context. Worldviews Evid Based Nurs 2011; 8: 15-24.

22. AACN, Standards for Establishing and Sustaining Healthy Work Environments. American Association of Critical-Care Nurses 2005. [Consultado 04-06-2012]. Disponible en http://www.aacn.org.

23. Bennet J, Dawoud D, Maben J. Effects of interruptions to nurses during medication administration. Nurs Manag 2010; 16: 22-23. 
24. Esi Owusu R, While A. Medication errors: types, causes and impact on nursing practice. Br J Nurs 2010; 19: 380-385.

25. FRY MM, DACEY C. Factors contributing to incidents in medicine administration. Part 1. Br J Nurs 2007; 16: 556-558.

26. McBride-Henry K, Foureur M. Medication administration errors: understanding the issues. Aust J Adv Nurs 2006; 23: 33-41.

27. Tissot E, Cornett C, Limat S, Mourand JL, Becker M, Etievent JP et al. Observational study of potential risk factors of medication administration errors. Pharm World and Sci 2003; 25 : 264-268.

28. TAXIS K, Barber N. Causes of intravenous medication errors: an ethnographic study. Qual Saf Health Care 2003; 12: 343-348.

29. Mayo AM, Duncan D. Nurse perceptions of medication errors: what we need to know for patient safety. J Nurs Care Qual 2004; 19: 209217.

30. DeAns, C. Medication errors and professional practice of registered nurses. Collegian 2005; 12: 29-33.

31. Westbrook JL, Wood A, Rob MI, Dunsmuir WTM, DAY RO. Association of interruptions with an increased risk and severity of medication administration errors. Arch Intern Med 2010; 170: 683-690.

32. Pape TM, Guerra DM, Muzquiz M, Bryant JB, InGram M, Schranner B et al. Innovative Approaches to reducing nurses' distractions during medication administration. J Contin Educ Nurs 2005; 36: 108-116.

33. Ebright P, Patterson E, Chalko B, Render M, MARTER L. Understanding the complexity of registered nurse work in acute care settings. J Nurs Adm 2003; 33: 630-638.

34. Myny D, Van Hecke A, De Bacquer D, Verhaeghe $\mathrm{S}$, Gobert M, Defloor T et al. Determining a set of measurable and relevant factors affecting nursing workload in the acute care hospital setting: A cross-sectional study. Int J Nurs Stud 2012; 49: 427-436.

35. BRUSH K. Upgrading systems design to reduce medication administration errors. Clin Nurse Spec 2003; 17: 15-16.

36. Bennet J, Harper-Femson L, Tone J, Rajmohamed Y. Improving medication administration systems: An evaluation study. Can Nurse 2006; 102: 35-39.

37. Potter PL, Wolf L, Boxerman S. Understanding the cognitive work of nursing in the acute care environment. J Nurs Adm 2005; 35: $327-$ 335 .
38. Rathmann KL, Meadows L, Simpson K. Creation of a "Patient Safety Zone" to reduce pharmacy and nursing distractions and improve patient care. 2007; 42: 2-6.

39. Palese A, Sartor A, Costaperaria G, Bresadola V. Interruptions during nurses' rounds in surgical wards: Observational study. J Nurs Manag 2009; 17: 185-192.

40. Poon EG, Keohane CA, Yoon CS, Ditmore M, Bane A, Levtzion-Korach O et al. Effect of barcode technology on the safety of medication administration. N Engl J Med 2010; 362: 16981707.

41. McRoberts S. The use of bar code technology in medication administration. Clin Nurse Spec 2005; 19: 55-56.

42. Englebright JD, Franklin M. Managing a new medication administration process. JONA 2005; 35: 410-413.

43. Perry S, Thamer M. Medical innovation and the critical role of health technology assessment. JAMA 1999; 282: 1869-1872.

44. McCoy LK. Look-alike, sound-alike, drugs review: include look-alike packaging as an additional safety check. Jt Comm J Qual Patient Saf 2005; 31: 47-53.

45. Schulmeister L. Look-alike, sound-alike oncology medications. Clin J Oncol Nurs 2005; 10: 35-41.

46. BERMAN A. Reducing medication errors through naming, labeling, and packaging. J Med Syst 2004; 28: 9-29.

47. Aibar-Remón C. El conocimiento del riesgo asistencial: ¿qué pueden aportar los pacientes? An Sist Sanit Navar 2012; 35: 5-8.

48. Mira JJ, Vitaller J, Lorenzo S, Royuela C, PérezJover V, Aranaz J. Pacientes como informadores de eventos adversos. Resultados en diabetes y enfermedad renal. An Sist Sanit Navar 2012; 35: 19-28.

49. Khun AM, YoungBerg BJ. The need for risk management to evolve to assure a culture of safety. Qual Saf Health Care 2002; 11: 158-162.

50. Lozano JM. De patos, gansos y cisnes. Revisiones narrativas, revisiones sistemáticas y meta-análisis de la literatura. Redalyc 2005; 30: 1-3.

51. Antman EM, Lau J, Kupelnick B, Mosteller F, Chalmers, TC. A comparison of results of meta-analyses of randomized control trials and recommendations of clinical experts. JAMA 1992; 268: 240-248.

52. Collins JA, FAuSER B. Balancing the strengths of systematic and narrative reviews. Human Reproduction Update 2005; 11: 103-104. 